\title{
RELATOS DE VIAJES: MAESTRAS, ESCUELAS Y CAMINOS RURALES EN EL MÉXICO DEL SIGLO XX
}

\author{
Blanca Susana Vega Martínez ${ }^{1}$ \\ www.psicologia.uaslp.mx \\ Norma Ramos Escobar² \\ www.upn.mx
}

\begin{abstract}
Resumen: En este trabajo exponemos los relatos de viaje de tres maestras rurales mexicanas que transitaron dentro del noreste del país para fundar escuelas y llevar a cabo su práctica docente. Las historias de maestras están inscritas en el México del siglo XX, el cual contaba con una población altamente analfabeta y que requería de una educación prioritaria. Las jovencitas casi niñas, llamadas "maestras empíricas" tuvieron que sortear diferentes vicisitudes para trasladarse en la región, implicando con ello dejar la vida familiar y autoconstruirse como mujeres trabajadoras que recorriendo caminos, comunidades y sierras pudieron cumplir con su labor docente, que era desde "hacer escuela" hasta curar enfermos. En este texto, reconocemos sus condiciones de trabajo, y desde su propia voz relatamos los viajes a la escuela y la comunidad, además de ello, contamos sus experiencias ante los constantes cambios de comunidades, el acceso a las escuelas y las precariedades de la educación rural. Con la responsabilidad de la familia y en un paralelismo de su trabajo y su vida cotidiana, los caminos de las maestras rurales fueron complejos y con condiciones adversas para las noveles maestras.
\end{abstract}

Palabras clave: Relatos de viaje; Maestras rurales; México; Siglo XX.

\section{NARRATIVAS DE VIAGEM: PROFESSORAS, ESCOLAS E CAMINHOS RURAIS DO MÉXICO NO SÉCULO XX}

Resumo: Neste trabalho analisamos os relatos de viagem de três professoras rurais mexicanas que transitaram dentro do nordeste do país para fundar escolas e levar adiante sua prática de ensino. As histórias das professoras estão inscritas no México do século XX, o qual contava com uma população altamente analfabeta e que requeria uma educação prioritária. As jovenzinhas, quase meninas, chamadas "professoras empíricas" tiveram que enfrentar diferentes vicissitudes para transitar na região, implicando com isso deixar a família e autoconstruir-se como mulheres trabalhadoras,

\footnotetext{
${ }^{1}$ Doctora en Humanidades. Universidad Autónoma de San Luis Potosí. Contacto: susanavega8@hotmail.com.

${ }^{2}$ Doctora en Humanidades. Universidad Pedagógica Nacional. Contacto: amronramos@gmail.com.
} 
que recorrendo caminhos, comunidades e serras puderam cumprir com seu trabalho que era desde "fazer escolas" até curar doentes. Neste texto, reconhecemos suas condições de trabalho e a partir de sua própria voz, relatamos as viagens à escola e à comunidade, além disso, contamos suas experiências frente às constantes mudanças de comunidades, o acesso às escolas e as precariedades da educação rural. Com a responsabilidade de sustentar a família e em um paralelismo do seu trabalho e de sua vida cotidiana, os caminhos das professoras foram complexos e com condições adversas para as professoras principiantes.

Palavras-chave: Narrativas de viagem; Professoras rurais; México; Século XX.

"Cuando alguien realiza un viaje, puede contar algo", reza el dicho popular, imaginando al narrador como alguien que viene de lejos. Pero con no menos placer se escucha al que honestamente se ganó su sustento, sin abandonar la tierra de origen y conoce sus tradiciones e historias.

Walter Benjamín

\section{Introducción}

Escribir sobre viajes implica reconocer las motivaciones del mismo y la diversidad de viajeros, entre las motivaciones por las que se emprende un viaje encontramos desde la búsqueda de conocimiento, el placer de la conquista, el exilio, la huida, el encuentro con el ser, la aventura, la exploración, el trabajo, y un sinfín de razones; con los viajeros no suele ser diferente, vemos al religioso, al médico, el antropólogo, al turista, al explorador, al peregrino, al migrante, al exiliado, y por supuesto al maestro rural, que pocas veces es reconocido como tal. A través de la narrativa de sus experiencias en la escuela o la comunidad, el maestro rural se convirtió en un viajero de aprendizajes y enseñanzas, aprendizajes con el otro, de su contexto y su ser, no solo el ser del otro sino el ser de sí mismo. Su enseñanza y aprendizaje se construyó a través de las comunidades que visitó y de las escuelas que erigió, de las casas de maestros que fundó, y las aulas que gestionó. Para el caso mexicano es preciso advertir que el 
magisterio es un campo históricamente feminizado (GALVÁN Y LÓPEZ, 2008), de ahí que centremos la mirada en las maestras rurales que se desempeñaron en las comunidades con múltiples tareas, que no solo tuvieron la de enseñar, sino que a la vez fungieron de enfermeras, campesinas, cuidadoras, y hasta veterinarias.

Los relatos que presentamos son historias de maestras rurales, las cuales fueron fruto de dos investigaciones llevadas a cabo en el noreste de México, dichas historias dan cuenta de las condiciones sociales y de trabajo que las maestras vivieron, desde sus experiencias nos encaminan a imaginar un contexto desfavorecido de la educación rural en México durante el siglo XX. Como un primer acercamiento exponemos el contexto general sobre las maestras rurales en México y posteriormente dejamos que sean las maestras las que cuenten sus relatos de viaje hacia las comunidades, el primer encuentro con la escuela rural, así como las maneras de llegar a ella. Como bien lo señala Guardia (2011, p. 27):

Las mujeres viajaron y mucho. Se aventuraron a través de territorios desconocidos por diversas razones: para esconderse, por compromiso, obligadas, por placer, para estudiar, y escribir. Viajes que no necesariamente significaron un desplazamiento geográfico, sino también una manera de transformar la ideología orientada a restringir su movilidad e independencia.

En este sentido, las maestras rurales fueron viajeras por preeminencia, aunque no hayan sido reconocidas como tal, en sus relatos nos muestran los (des) encuentros con las comunidades y por supuesto con los viajes hacia la escuela.

\section{Maestras rurales en México: enseñanzas y aprendizajes en el camino}

Los cambios sociodemográficos de los maestros a lo largo del siglo $\mathrm{XX}$ se vieron reflejados a decir de Arnaut en tres vertientes:

En el siglo XIX y hasta la tercera década del siglo XX, la mayoría de los maestros prestaba sus servicios en las zonas urbanas y 
semiurbanas del país; a partir de 1920 ganan presencia los maestros rurales; de 1930 a 1960 aproximadamente, el mayor número de los maestros prestaban servicios en zonas rurales y semiurbanas; finalmente, a partir de 1960 predominan en el magisterio los maestros que trabajan en las zonas urbanas y semiurbanas. (ARNAUT, 2012, p. 197)

De estos maestros rurales que nos señala el autor, cabe decir que gran parte fueron maestras, es decir, el magisterio del que se habla era propiamente femenino, ellas al igual que los maestros rurales tuvieron un papel relevante en las comunidades. Ya algunos autores señalan la diversidad de tareas que tenían a su cargo, pero también su involucramiento con la comunidad y sus miembros. Loyo (1990, p. 328) lo advierte:

El maestro que trabajaba en el campo y en los barrios marginados no limitaba su tarea a la simple instrucción rudimentaria. Su labor iba más allá del aula escolar, abarcaba a toda la comunidad, niños y adultos por igual, y su objetivo era ayudarlos a llevar una vida mejor en todos los órdenes. Sus enseñanzas incluyeron la castellanización, un mejor aprovechamiento de los recursos naturales de la región, la comunicación de los grupos aislados y el mejoramiento de la vida doméstica. Compartir la vida ardua y miserable del trabajador convirtió al maestro no solo en su más fiel aliado y compañero sino también en un líder que le señalaba sus derechos y lo inducia a pelear por ellos.

Es por ello, que las maestras rurales del siglo XX fueron parte fundamental en la educación, en el proyecto de nación y en los cambios sociales, políticos y culturales de la época.

La escuela rural se diseñó bajo la idea de convertirse en el centro regulador de la vida de las familias campesinas, luego de la Revolución Mexicana una de las demandas principales de los campesinos fue educación, de ahí que la política del estado posrevolucionario fue la expansión de la escuela rural, por ello la necesidad de reclutar maestras y maestros para alfabetizar a la gran parte de la población campesina, que para entonces era de mayores porcentajes en México, un país que despuntaba el siglo XX con un 80\% de analfabetismo. Fue así que los pocos y pocas que sabían leer y escribir fueron los primeros maestros y maestras cualificados como "empíricas(os)", lo que determinará las frágiles condiciones laborales del magisterio mexicano. López (2001) ha identificado que pos- 
terior a la creación de la Secretaria de Educación Pública en 1921, en México se da la entrada masiva de las mujeres al magisterio, ante el llamado que hiciera José Vasconcelos (primer Secretario de Educación) para invitar a la mujer mexicana a alfabetizar, y es a partir de entonces que se construye un imaginario sobre "la maestra rural".

\section{Condiciones sociales y de trabajo}

La primera oleada de maestras rurales que feminizaron el magisterio mexicano en las primeras décadas del siglo XX compartía algunas características: eran mujeres que apenas habían terminado la primaria elemental (cuarto año), las menos la primaria superior (sexto año) y muy pocas eran normalistas (RAMOS, 2007; LÓPEZ, 2008). Sus condiciones laborales fueron precarias, sus sueldos eran menores al de sus compañeros varones, justificados por las autoridades educativas y gubernamentales como sueldos honorarios o "gratificaciones pequeñas", por considerar que eran "hijas de familia" que no necesitaban el sustento (RAMOS, 2007, p. 149). Otra de las características compartidas era que en su mayoría eran jóvenes y solteras (RAMOS, 2007; LÓPEZ, 2001, 2008; ROCKWELL, 2008). Su trabajo en las zonas rurales fue mejor acogido debido a que en su mayoría las escuelas rurales eran mixtas debido a las condiciones económicas que no permitían fundar escuelas para niños y niñas, además que la política educativa posrevolucionaria incentivó la coeducación y las comunidades rurales preferían tener una mujer como maestra. Este es el escenario que envolvía las trayectorias profesionales y la movilidad espacial de las maestras rurales. En síntesis, las condiciones de las maestras rurales no eran las más favorecidas, sin una preparación profesional que ayudara en su práctica, sin los recursos necesarios en las comunidades, en muchas de las ocasiones sin un lugar idóneo para impartir enseñanza, con sueldos que solo les permitían vivir con austeridad y/o precariedad en sus lugares de vivienda, sino que, además siendo demasiado jóvenes, tuvieron la tarea de modernizar a las comunidades campesinas. 
Como lo reafirma también Castillo (2010) por más de cinco décadas las maestras rurales trabajaron en espacios escolares improvisados poniendo a prueba su interés al contar con un empleo mal remunerado, pero con la posibilidad de mejorar sus condiciones de vida ya de por sí modestas.

Las maestras entrevistadas no fueron la excepción, una de ellas así lo relata: "almorcé y me fueron a mostrar la escuela, la escuela era únicamente el salón, no había bancos, pizarrón, nada [...] De rato llegó un señor con un pedazo de lona negra y un gis, lo clavó y empecé" (HERNÁNDEZ, 2011). Por su parte la maestra Francisca (2005) lo relata así:

Empecé a trabajar con los niños debajo de los árboles, no había salón, iPuro árbol! Llovía y corríamos a la primera casa que nos dieran alojo mientras pasaba el agua. Había comisiones de niños, y a los que les tocaba la comisión del pizarrón corrían para protegerlo de la lluvia, y el resto con las sillas, que no eran más que pedazos de palma seca.

Además de las condiciones sociales desfavorecidas, los maestros rurales vivieron con grandes peligros por ser reconocidos como líderes campesinos. Raby (1968, p. 191) nos señala "su participación en los conflictos sociales y sus conexiones políticas no se revelan fácilmente en los archivos y en los periódicos de la época; pero se sabe bien que los años que nos interesan se asesinaban, desorejaban, o en otras maneras se hostilizaban a los maestros rurales". Y con las maestras rurales las cosas no fueron muy diferentes, fueron objeto de ultrajes, abusos, violaciones, y robos en las comunidades. El mismo autor nos señala casos de maestras secuestradas, ultrajadas y asesinadas en las comunidades, es por ello que muchas de las maestras rurales acostumbraban a viajar con acompañantes: madres o hermanos, y en algunos casos, recibían la visita constante de su esposo o familia. En este caso, la maestra Petra lo relata:

Como uno era muy joven tenía muchos pretendientes, ahí era donde a mí me daba miedo, mandaban cartas, y ahí si me daba miedo, porque algunas [maestras] si les había pasado que a fuerza las agarraban. El municipio de Villa de Guadalupe era muy conocido porque a las maestras se las llevaban a la fuerza, las violaban, y no los metían a la cárcel porque se casaban con 
ellas, ipero cómo se iban a casar con un muchacho que ni conocían ni platicaban! Pero así era, los muchachos decían me gusta la maestra, la agarro y me la llevo, como si fuera una cosa fácil. (PETRA, 2011).

Por otra parte, también existían intereses por parte de las autoridades escolares, y amenazas de separación de cargo, sino se cumplía con los mandatos en las comunidades "se va a donde la mande o sino renuncia".

De aquí que las maestras pueden verse como sujetos históricos, $\mathrm{y}$ sus desplazamientos como viajes de aprendizaje y enseñanza. Pero no podemos negar que sus viajes fueron también promovidos o negados por el mismo sistema educativo, por ejemplo, en las zonas "peligrosas", "conflictivas" o "lejanas" los inspectores escolares preferían mandar un maestro varón en la idea que por su condición de hombres podían enfrentarse con agraristas o campesinos, como en el Valle del Mezquital que relata López (2001), o en el caso de Tlaxcala que narra Rockwell (2008), de ahí que la migración femenina responda también a criterios de salvaguardar la integridad de las mujeres, su migración fue más regional-municipal.

\section{Historias de maestras rurales. Viajes entre comunidades rurales del Altiplano en México}

Como lo hemos comentado, las mujeres han caminado por varios senderos, su desplazamiento del espacio privado al espacio público representó una nueva forma de posicionarse en el mundo, se aventuraron a lo desconocido, y la educación fungió como medio para reconocerse como sujetos con agencia. Su profesionalización fue una manera de viajar, en ella, pudieron encontrarse y reinventarse, para las maestras rurales, ésta les permitió no solo iniciarse y/o formarse como maestras sino que también implicó una serie de cambios personales, como dejar a la familia, su lugar de origen, desplazarse cada semana o mes de visita a casa, así como tener condiciones precarias en sus lugares de trabajo.

El contexto de las maestras de las que hablamos es la altiplanicie mexicana al noreste del país, zona que se encuentra en promedio a 1.500 


\section{Revista de História e \\ Historiografia da Educação}

metros sobre el nivel del mar, el clima oscila entre los 30 y 40 grados, también catalogada como zona semidesértica, de poca agua, de ahí su precaria agricultura, es de mayor importancia su producción ganadera caprina y una destacada producción agro-industrial como la producción de ixtle3.

Foto 1 - Maestros en prácticas agrícolas. Dr. Arroyo, Nuevo León, AHSEP/Fondo Misión Culturales, Caja: 82. Exp. 1, 1928.

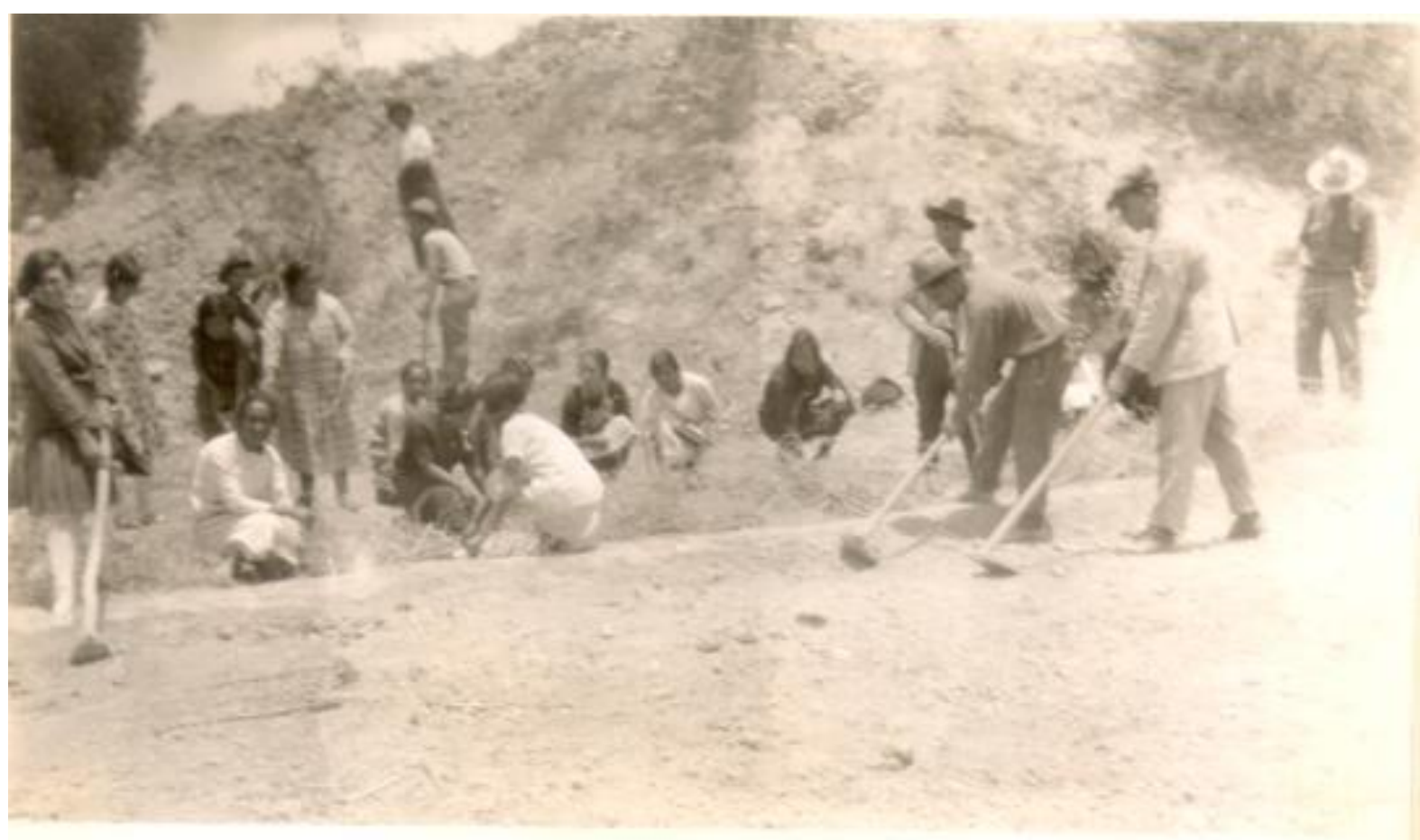

En este texto mostramos los relatos de viaje de tres maestras rurales del noreste de México, sus experiencias nos dan muestra de sus condiciones sociales y de trabajo, que les implicaba moverse constantemente de una comunidad a otra, nos muestran además los caminos hacia la escuela y los senderos por los que atravesaron para llegar a ella.

3 Fibra que se produce de cualquier agave, que sirve para hacer mecates, tejer cestas, bolsas para transportar, entre otras. 


\section{Primer viaje a la escuela}

El primer encuentro con la escuela y la comunidad representó para las maestras rurales un viaje por lo desconocido y el inicio de una aventura, a pesar de su corta edad, las maestras rurales se aventuraron por un trabajo y una labor que desconocían, tal como le sucedió a la maestra Petra en el Ejido La Gavía (Matehuala, San Luis Potosí) a la edad de 18 años:

Le dije a mi papá [que se iría como maestra] y me dijo que no porque en los ranchos 4 se sufría mucho, se batallaba, que era muy lejos, que yo no sabía adónde me iban a mandar, le dije que me dejara ir una semana, al final me dejaron pensando que me iba arrepentir, mi hermano el más chico se fue conmigo, yo tenía 16 años. Me llevaron con el inspector profesor Julián Martínez de la campaña alfabetizadora que era un programa federal, me dio algunas instrucciones y me fui, llegué a los mesones, donde llegaban las carretas, los carretones, ahí estaba un señor con dos burros esperando por mí. Me subí y yo iba arrastrando los pies, y le dije al señor que porque yo iba arrastrando los pies en la tierra, revisó y me dijo icómo no ha de ir arrastrando los pies si va en el pescuezo del burro! (risas). (HERNÁNDEZ, 2011).

La maestra Francisca narró cómo fue su primer desplazamiento a la comunidad de San José de Flores (Dr. Arroyo, Nuevo León), apenas cumplía los 14 años:

Al terminar el examen (sexto año en la cabecera municipal de Dr. Arroyo) las autoridades me felicitaron, se acercó el director de las escuelas rurales y el inspector, me dijeron "como ya no va a ver aquí secundaria, ya puedes ir a trabajar a una escuela rural como maestra, ¿qué te parece?, te vamos a pagar". Me dieron el nombramiento y fueron a casa a hablar con mis papás [...] Fue en 1928 cuando me asignaron el nombramiento en San José de Flores, tenía 14 años. Mi mamá me acompañó al ser yo la única mujer y la mayor. (RUIZ, 2005).

4 Lugar fuera de poblado, donde se albergan diversas familias o personas (Real Academia Española) 
La maestra Francisca recordó que llegaron a caballo y que el camino era desolado, aunque la comunidad se encontraba cerca de la carretera principal que llevaba a la cabecera municipal.

\section{Maneras de viajar}

Las maestras rurales viajaron de diversas maneras hacia la escuela y las comunidades, muchas veces las caminatas de varios kilómetros eran su cotidianeidad semanal o mensual. En otras, los propios padres de familia de la comunidad se organizaban para dejarlas en el municipio más cercano:

Entonces empecé a buscar como se iba uno, como se regresaba, a poco todo el tiempo la gente va y viene, pues sí hay puros animales, el medio de transporte era el carretón, el burro, ahí no había carreta, nomás carretones y carros ballesta que era largo y tenía cuatro ruedas. (HERNÁNDEZ, 2011).

En el caso de la maestra Francisca, el tránsito era a caballo, partían de la cabecera municipal donde vivía su familia y se desplazaban hasta las comunidades, su papá y hermanos la visitaban en San José.

Con la maestra Gela, la situación fue diferente, se casó con un maestro rural en la zona de Dr. Arroyo, la Secretaría de Educación Pública favoreció el matrimonio entre maestros, permitiéndoles estar en la misma escuela y compartir la casa para el maestro, lo que hacía fácil también moverlos cuando había una escuela que necesitara el servicio de dos docentes, así describe el cambio de escuela:

Entonces nos mandaron a los dos a San Ramón y luego al Tecolote, un lugar muy lejos, casi en el cerro. Gustavo [su marido], era muy listo para todo, compró un carro viejo que tenía muy buenas ruedas, le puso su asiento con su toldo y en ese nos íbamos con un par de mulas que lo estiraban. Así seguimos trabajando en las escuelas. (ÁLVAREZ, 2005). 


\section{Difícil acceso a las escuelas}

Llegar a las comunidades y escuelas durante las primeras décadas del siglo XX seguía siendo difícil, como lo señalamos, los medios de transporte eran escasos y muchas de las veces representaban tiempo, dinero y organización.

Los cambios de una comunidad a otra cada dos o tres años significaba reorganizar a la familia, replantear nuevas rutinas y cotidianeidades, y volver a conocer y adaptarse a las costumbres de la comunidad. La maestra Petra lo señaló así:

Cuando ya empecé a trabajar, me cambiaron a otro ranchito, ahí si había lo necesario para trabajar, la gente estaba muy interesada en que aprendieran los niños, yo me iba en un camión que iba hasta Dr. Arroyo, los lunes a las 2 de la tarde y de ahí caminaba como 1.5 kilómetros para llegar a la escuela, pero no iba y venía todos los días, ahí me quedaba de lunes a viernes, fue en 1958, ese año llovió mucho y se trabajó la parcela, sacamos bastante maíz. (HERNÁNDEZ, 2011).

Las permutas y cambios constantes de comunidades les representaron a las maestras una forma de vivir, no solo de trabajo, ya que muchas de ellas formaron familia con miembros de las comunidades, sin embargo, los peligros seguían latentes en cada camino a la escuela:

Me mandaron a San Pablo, pero salió igual, más bien permuté otra vez, me iba a Matehuala y de ahí eran 7 kilómetros, a veces iban por mí y otras veces no, entonces me iba caminando, se usaba que los padres de familia me llevaban a la cabecera municipal a tomar el camión, y otro padre me esperaba el lunes, pero si por alguna razón no llegaba había que irse a pie. Ahora pienso en todo aquel monte, caminando, y yo mirando el monte, si ahora estuviera joven no me iría (risas), no caminaba, que me fuera saliendo alguien y me golpeara, gracias a Dios nunca me pasó nada, pero yo creo que ahora no caminaba (HERNÁNDEZ, 2011).

El tránsito de las maestras por las comunidades también tenía que ver con su buen desempeño, estrategia que muchas de las veces eran im- 
plementadas por los inspectores. Así le ocurrió a Francisca, quien de trabajar muchos años en Dr. Arroyo fue trasladada a Aramberri, Nuevo León (municipio colindante con Dr. Arroyo) y el acceso a la comunidad fue más complicado:

Me comisionaron a fundar escuelas en Aramberri, las características eran muy diferentes a Dr. Arroyo, las comunidades estaban internadas en la sierra madre, había mucha vegetación, irrigación de ríos. Recuerdo que cuando iba cruzando a caballo por la sierra me tocó ver un tigrillo, había animales salvajes. Me dio tristeza ver que la gente vivía en cuevas de los cerros, en esas comunidades les llevaba hasta dos días ir a enterrar los cadáveres y llevar los enfermos. Había que empezar por hacer caminos. Empecé por organizar juntas con las familias, y como estas juntas eran de noche, por los caminos venía la gente alumbrándose con antorchas, lo difícil fue convencer a la gente para que dejaran de vivir en las cuevas y aprovecharan los recursos que tenían, había naranja, nuez, manzana... Allá fundé escuelas en San Juanito, Puerta de Abajo, los Álamos, San Rafael del Llano... (RUIZ, 2005).

\section{Dejar la comunidad}

Dejar la comunidad e ir a otra tenía diferentes motivaciones o causas, algunas de ellas venían directamente de las autoridades, y otras tenían que ver con los intereses o seguridades de las maestras rurales, por ejemplo, la distancia hacia sus hogares, la comodidad o incluso la idiosincrasia del pueblo.

Yo me cambiaba de comunidad constantemente porque veía algunas maestras que pasaban mucho tiempo en un lugar, conocí a una que duró 11 años en la comunidad y la gente se expresaba muy mal de ella, por eso no se debía durar mucho, unos 3 o 4 años y cambiarse, no todas las comunidades son iguales, cada comunidad es diferente, la gente es diferente [...] Se debe tomar en cuenta no dejarse absorber por la comunidad y prepararse constantemente, por eso yo me movía, a veces por eso, y otras porque me peleaba con la gente, porque yo no me dejaba, yo nunca me dejé en los ranchos, yo empecé muy chiquilla a traba- 


\section{Revista de História e

jar y a veces digo ¿cómo me enfrenté a gente que jamás había conocido, a sus costumbres?” (HERNÁNDEZ, 2011).

Foto 2 - Escuela Primaria Oficial "Benito Juárez", Buenavista, Matehuala, 1966. Archivo personal de la maestra Petra Hernández.

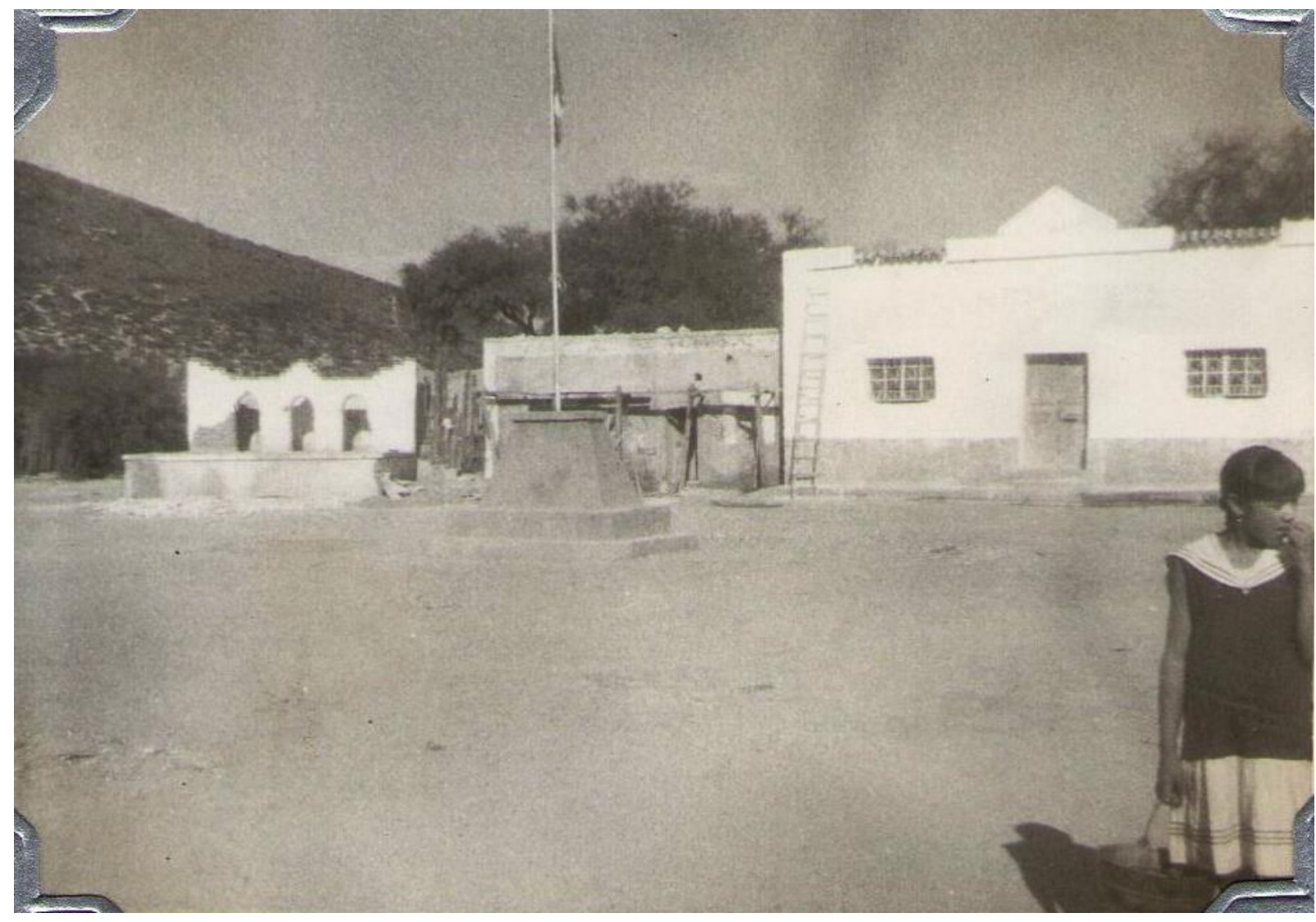

Las maestras no siempre estuvieron listas ni contentas de irse de la comunidad, como mencionamos, el cambio dependió de decisiones administrativas y políticas, pero el hacer maletas, trasladarse y empezar de nuevo resultó en ocasiones molesto para las maestras, así lo recuerda la maestra Francisca de su cambio de El Charquillo a San Pedro de la Rueda:

Como cada año había cambios, me trasladaron nuevamente a otra escuela. Por eso dice la gente: "maestra a usted le robaron, porque hizo usted todo el trabajo y luego la cambiaron"; y así fue, llegó un político con su esposa y se quedaron en mi lugar. El inspector me mandó a la sierra, a la escuela de San Pedro de la 
Rueda, no había nada, ni caminos vecinales ni nada entre la sierra, subía uno a caballo. Estuve dos años ahí, luego me transfirieron a la escuela de Medina, allá empecé [haciendo] con las carreteras... (RUIZ, 2005).

La maestra Gela describe así sus últimos recorridos en las escuelas rurales:

Viví muy a gusto en el campo. Las comunidades eran muy pobres, pero recibimos muchos favores de los campesinos nos llevaban elotes, quelites, nopales, pollo, cabrito... Cuando nos sacaron de la escuela de El Tecolote, Gustavo ya era secretario de la Sección 21 del Sindicato. El juez auxiliar del Tecolote lloró mucho porque sacaron a Gustavo, él era que los dirigía en la cuestión agraria. Acabábamos de formar la plaza de armas del lugar cuando nos fuimos a Monterrey. (ÁLVAREZ, 2005).

\section{Para concluir: con la responsabilidad de la familia y el hogar}

Las migraciones de las maestras rurales tuvieron implicaciones en su vida que poco se han registrado, al igual que las mujeres de los ejércitos revolucionarios, el desplazamiento de las maestras significó llevar la domesticidad en hombros. Las entrevistadas señalaron que tuvieron que criar a los hijos en las escuelas rurales, Francisca comentó "cuando nació mi hijo Emigdio Javier, me lo llevaba en un cajón con ruedas a la escuela, él creció en las escuelas donde yo iba”. La maestra Petra también lo vivió:

Se trabajaba en un cuarto de una hacienda, si tenía salón de clases y cuarto de maestro, pero los cuartos se estaban cayendo. Yo ya tenía a uno de mis hijos. Mi hijo estaba chiquito, el salón estaba cerca del cuarto donde nos quedábamos y al lado estaba la cocina. Cuando él estaba acostadito y lloraba, iba y lo veía, si veía que lloraba porque tenía hambre, le preparaba su alimento y aprovechaba la hora de receso para cuidarlo. (HERNÁNDEZ, 2011).

Los largos viajes a caballo en los caminos y rancherías durante muchos años, pudo haber traído como consecuencia que sus embarazos fueran riesgosos e incluso no logrados, así lo narró la maestra Francisca: 
Cuando una anda a caballo el trote descompone la matriz, ya no tuve más hijos, después ya no quise, porque o te quedas a cuidar a la familia o a trabajar... yo no quería cortar mi carrera, pensaba en mi pensión. (RUIZ, 2005).

Si bien la maestra Gela no relacionó sus abortos a los peligros del trote y de los viajes, sí vivió situaciones difíciles:

No tuve hijos, tuve tres abortos y luego ya no volví a embarazarme. Recuerdo que el doctor Tamayo me revisó dijo que no sabía por qué abortaba, que estaba perfectamente bien mi matriz, que estaba bien lista para tener familia y que no se explicaba por qué tenía abortos (ÁLVAREZ, 2005).

Las maestras no podían arraigarse en un sitio, muchas de ellas tuvieron casa fija y propia hasta que les faltó poco para jubilarse o bien cuando llegaron y se establecieron en las cabeceras municipales, pero lo hicieron cuando los hijos crecieron y tuvieron la necesidad de enviarlos a estudiar a las escuelas de nivel bachillerato y estudios superiores, o bien porque la carrera de sus maridos implicó trasladarse a la ciudad.

Los viajes de las maestras rurales representaron una difícil decisión en sus vidas, ya que no solo era transitar entre comunidades por razones de trabajo, sino una forma de vida; desplazarse dos o tres veces por mes a "casa" implicó que no reconocieran a la comunidad como su hogar -salvo excepciones, es decir, que formaran familia con miembros de la misma comunidad-, sino como un lugar de paso, un lugar en el que aguardaban mientras llegaba otra oportunidad de trabajo con mejor condiciones de vida.

Con ello quizá tendríamos que pensar en los significados que para las mujeres implicó viajar, pero también lo que representó dejar de hacerlo. 


\section{Referencias}

ARNAUT, A. Los maestros de educación primaria en el siglo XX, In: LATAPÍ, P. Un siglo de educación en México, v. II. México: Fondo de Cultura Económica, 2012

CASTILLO, S. Sembradoras del saber. Estado de México: Servicios Educativos Integrados al Estado de México (SEIEM), 2011.

GALVÁN, L. E.; LÓPEZ, O. Entre imaginario y utopías: historias de maestras. México: Publicaciones de la Casa Chata - CIESAS/Colsan/ PUEG, 2008.

GUARDIA, S. Viajeras entre dos mundos. Centro de Estudios la Mujer en la Historia de América Latina CEHMAL, 2011.

LÓPEZ, O. Alfabeto y enseñanzas domésticas. El arte de ser maestra rural en el Valle del Mezquital. México: CIESAS/Consejo Estatal para la Cultura y las Artes en Hidalgo, 2001.

LÓPEZ, O. Porfirianas y revolucionarias: dos estudios de caso de maestras mexicanas, In: GALVÁN, L. E.; LÓPEZ, O. Entre imaginario y utopías: historias de maestras. México: Publicaciones de la Casa ChataCIESAS/Colsan/PUEG, 2008.

LOYO, E. Escuelas rurales "Artículo 123" (1917-1940). Historia Mexicana, v. 40, n. 2, 1990.

RABY, D. L. Los maestros rurales y los conflictos sociales en México (1931-1940). Historia Mexicana, v. 18, n. 2, 1968.

RAMOS, N. El trabajo y la vida de las maestras nuevoleonesas. Un estudio histórico de finales del siglo XIX y principios del XX. Monterrey: CONARTE, 2007.

ROCKWELL, E. Las maestras en Tlaxcala antes y después de la Revolución, In: GALVÁN, L. E., LÓPEZ, O. Entre imaginario y utopías: historias de maestras. México: Publicaciones de la Casa Chata - CIESAS/ Colsan/PUEG, 2008.

\section{Fuentes orales}

ÁLVAREZ, M. Á. Entrevistas realizadas con la maestra por Norma Ramos Escobar, durante el mes de julio de 2005 en el municipio de Dr. Arroyo, Nuevo León, México. 
HERNÁNDEZ, F. Entrevistas realizadas con la maestra por Norma Ramos Escobar en el mes de abril de 2005, en la ciudad de Monterrey, Nuevo León, México.

HERNÁNDEZ, P. Entrevistas realizadas con la maestra por Blanca Susana Vega Martínez durante los meses de octubre de 2011 a marzo del 2012, en la ciudad de San Luis Potosí, S.L.P., México.

Recibido el 12 de marzo de, 2017. Aprobado el o3 de abril 2017. 